\title{
Forces, Stresses and the (Thermo?) Dynamics of Active Matter
}

\author{
Sho C Takatori and John F Brady* \\ Division of Chemistry and Chemical Engineering, California Institute of Technology, Pasadena, California 91125, USA \\ *jfb@cheme.caltech.edu
}

\begin{abstract}
The statistical mechanics and microhydrodynamics of active matter systems have been studied intensively during the past several years, by various soft matter physicists, chemists, engineers, and biologists around the world. Recent attention has focused on the fascinating nonequilibrium behaviors of active matter that cannot be observed in equilibrium thermodynamic systems, such as spontaneous collective motion and swarming. Even minimal kinetic models of active Brownian particles exhibit self-assembly that resembles a gas-liquid phase separation from classical equilibrium systems. Self-propulsion allows active systems to generate internal stresses that enable them to control and direct their own behavior and that of their surroundings. In this Review we discuss the forces that govern the motion of active Brownian microswimmers, the stress (or pressure) they generate, and the implication of these concepts on their collective behavior. We focus on recent work involving the unique 'swim pressure' exerted by active systems, and discuss how this perspective may be the basic underlying physical mechanism responsible for self-assembly and pattern formation in all active matter. We discuss the utility of the swim pressure concept to quantify the forces, stresses, and the (thermo?) dynamics of active matter.

Keywords: active matter; swim pressure; nonequilibrium systems; collective behavior
\end{abstract}

\section{Introduction}

A distinguishing feature of many living organisms is their ability to move, to self-propel, to be active. Constituents of "active matter" systems are capable of independent self-propulsion by converting fuel into mechanical motion, and include both microscopic entities like microorganisms and motor proteins within our cells to large bodies like fishes and birds. Inanimate, nonliving bodies can also achieve self-propulsion using mechanisms that are different than living organisms, but the outcome of their collective behavior is not necessarily different between living and nonliving active systems. Indeed, active matter systems of all scales have the tendency to associate together and move collectively, from colonies of bacteria, swarms of insects, flocks of birds, schools of fish, and herds of cattle. A question arises as to the micromechanical origin for living organisms to exhibit collective and coherent motion, and whether it can be explained and expressed using basic physical quantities.

All active matter systems are intrinsically out of equilibrium, a trait which allows self-propelled entities 
to display fascinating behaviors that cannot be observed in thermodynamic systems in equilibrium such as spontaneous self-assembly and pattern formation 1, 2, 3, 4, At the same time, nonequilibrium systems like active matter have very complex and specialized networks relating the input to the response, which make the theoretical understanding of their behaviors a challenging and intriguing problem in soft matter and statistical mechanics.

In this Review, we discuss the unique forces, stresses, and (thermo)dynamics of active matter, in an effort to offer a simple perspective to help explain many of the intriguing behaviors exhibited by active systems. In particular, we focus on a new 'swim pressure' principle [5, 6] to explain the self-assembly of active particles and analyze the applicability of conventional thermodynamic concepts to a nonequilibrium system. First, in Sec 2 we review the different classes of active swimmers including both living microorganisms and inanimate, synthetic self-propelled entities, and the methods to control and manipulate their motion. Next, we discuss the forces that govern the motion of microswimmers at low Reynolds number, explaining why and how microorganisms are able to move while being 'force-free.' Because of their ability to self-propel and reorient, active swimmers have an enhanced effective translational diffusivity, which we explain in Sec 4 . Owing to the swimmer's tendency to wander away and diffuse, a swimmer enclosed inside a container would exert a pressure on the surrounding boundaries of the container as it interacts with the walls. As described in Sec 5. this is the physical origin of the 'swim pressure,' a unique mechanical pressure generated by all active systems as a result of their self-motion. In Sec 6 we consider different approaches in the literature that have analyzed the fascinating collective behavior exhibited by active matter. We focus on the swim pressure perspective to predict the onset of phase separation in a simple active Brownian suspension. Next, in Sec 7 we discuss whether the notion of an effective 'temperature' of active matter can be used to describe the activity of an active suspension. In Sec 8 we explain of how active systems may exert an 'internal' force that behaves just like an external body force like gravity and how one can describe active systems from a microscopic theory. Finally, in Sec 9 we conclude with suggestions for future research.

\section{Active matter systems}

Among a large class of active matter systems, we turn our attention on microscopic swimmers whose size $a$ and swimming speed $U_{0}$ are such that the Reynolds number associated with their self-motion is negligibly small $R e \equiv U_{0} a / \nu \sim \mathcal{O}\left(10^{-6}-10^{-2}\right)$ ( $\nu$ is the fluid kinematic viscosity, taken to be that of water), and hence their dynamics are governed by the Stokes equations. Unlike large organisms like fish that self-propel by making use of inertia in the surrounding fluid, bodies at low-Reynolds number must break time-reversal symmetry to move. Active matter systems need not be living, and in fact intensive research has gone into the fabrication of nonliving, synthetic microswimmers, as described below. 


\subsection{Inanimate, synthetic particles}

Despite a recent increase in active matter research, fabrication of artificial systems of autonomously moving and self-assembling bodies have been done for some time. Purcell's three-link swimmer [7] and Taylor's toroidal swimmer [8] are a few early examples which illustrate the importance of breaking symmetry to move in the Stokes regime. An asymmetric chemical reaction occurring around a body's surface also results in self-motion from symmetry breaking; a famous example is camphor crystals placed on an water/air interface that self-propel by an asymmetric dissolution of camphor creating surface tension gradients that induce motion [9. Aside from gradients in surface tension, Ismagilov et al[10] fabricated a micro-plate that self-propelled on a water surface by having an asymmetric coating of platinum that reacted in a solution of hydrogen peroxide. These plates moved by releasing bubbles generated by the decomposition of hydrogen peroxide at the platinum surface. Others [11, 12] created micron-sized particles that had half of the particle surface coated with a thin layer of platinum. Called 'Janus particles,' only the areas coated with platinum decompose hydrogen peroxide, which result in an asymmetric distribution of reactants and products along their surface. In self-electrophoresis [13, 14, bimetallic particles composed of two different metals (e.g., $\mathrm{Au} / \mathrm{Pt}$ ) can generate self-propulsion due to an ionic current resulting from a difference in electron affinities of the two metals. In self-diffusiophoresis [15], autonomous motion is attributed to the osmotic pressure gradient induced by an asymmetric distribution of solutes around the particle[16]. However, the full mechanism behind the motion of self-diffusiophoretic particles is not fully understood [17]. Nonetheless, Janus microswimmers have become a standard model for active Brownian colloids and are used frequently by researchers around the world.

Active Janus particles swim roughly at a fixed speed $U_{0}$ in a direction specified by a body-fixed unit orientation vector $\boldsymbol{q}$, as shown in Fig 1 . The orientation $\boldsymbol{q}$ changes by Brownian motion with rotational diffusivity $D_{R}$ so the Janus particle has a characteristic reorientation timescale given by $\tau_{R} \sim 1 / D_{R}$.

Light-activated Janus particles [18, 19, 20] offer a convenient method to control the speed $U_{0}$ of the particles - the chemical reaction taking place at the particle surface is trigged by light, which allows researchers to instantly turn on or off the particle motion. Researchers have also fabricated Janus motors with layers of ferromagnetic material that allow for magnetic alignment of the swimmer orientation to move in a directed fashion [21], providing a method to control the reorientation time $\tau_{R}$ in addition to the speed $U_{0}$.

\subsection{Living microorganisms}

In addition to artificial microswimmers, another large class of active matter include living microorganisms, which can be divided into 4 sub-categories based upon their swimming mechanism: ciliates such as Paramecium mobilize small flagella around their body; flagellates such as E. coli activate a single or multiple flagella; spirochetes such as Leptospira use axial filaments to undergo a twisting, corkscrew motion; and amoebas such as Amoeba proteus deform their entire body. Many motile microorganisms like E. coli undergo a run-and-tumble where they alternate between a "run," where they swim straight towards a given 
orientation with speed $U_{0}$, and a "tumble" which aligns them into a new random direction with frequency $\omega[22$. Like a synthetic active Janus particle, the activity of a motile microorganism may be described by an average speed $U_{0}$ and reorientation time $\tau_{R} \sim 1 / \omega$.

Similar to synthetic particles that allow for magnetic control, external fields like chemical and thermal gradients, and magnetic and gravitational fields can cause living microorganisms to modify their swimming behavior to facilitate movement to a favorable region. E. coli have been known to undergo chemotaxis by preferentially swimming towards (or away from) chemical gradients of nutrients (or toxins) [23]. Magnetotactic microorganisms such as Magnetospirillum have organelles called magnetosomes that contain magnetic crystals that help the organism align along imposed magnetic field lines 24]. Other common examples of taxis swimmers include phototactic[25] and gravitactic[26] bacteria. Chlamydomonas reinhardtii is a green alga that swims with a breast-stroke motion and possesses an eyespot that allows the alga to orient itself and swim toward a light source 27.

Now that we have briefly reviewed the different classes of microswimmers, in the next section we discuss the self-propulsive forces that govern their motion in the Stokes regime, explaining why and how microorganisms are able to move while being 'force-free.'

\section{3. 'Force-free' motion}

Swimming microorganisms and inanimate self-propelled particles move in the Stokes regime and undergo so-called 'force-free' motion. This phrase is somewhat ambiguous, as all non-accelerating bodies are by definition force-free, i.e., $m(d \boldsymbol{U} / d t)=\sum \boldsymbol{F}=\mathbf{0}$. This is true for an airplane traveling at constant speed, where its propulsive force is balanced by the frictional forces acting against the body. The same applies for microscopic bodies swimming in low-Reynolds number. What researchers actually mean when they say active matter undergoes force-free motion is that the body experiences no external force causing the body to move.

What force then, if any, induces the body to swim? Suppose we have a ciliated microorganism (such as Paramecium) which swims by beating many small flagella cooperatively along its body surface, such that the velocity of the surrounding fluid at any point on the swimmer surface is $\boldsymbol{u}(\boldsymbol{x})=\boldsymbol{U}+\boldsymbol{\Omega} \times(\boldsymbol{x}-\boldsymbol{X})+\boldsymbol{u}^{s}(\boldsymbol{x})$, where $\boldsymbol{U}$ and $\boldsymbol{\Omega}$ are translational and angular velocities of the body (about its center), $\boldsymbol{X}$ is the position of the body center, $\boldsymbol{x}$ is the position along the body surface, and $\boldsymbol{u}^{s}(\boldsymbol{x})$ is the 'slip velocity' induced by the deformations happening along the ciliated body surface. The slip velocity can be expressed in terms of surface moments: $\boldsymbol{u}^{s}(\boldsymbol{x})=\boldsymbol{E}^{s} \cdot \boldsymbol{x}^{\prime}+\boldsymbol{B}^{s}:\left(\boldsymbol{x}^{\prime} \boldsymbol{x}^{\prime}-\boldsymbol{I}\left(x^{\prime}\right)^{2}\right)+\cdots$, where $\boldsymbol{x}^{\prime}=\boldsymbol{x}-\boldsymbol{X}$, and the surface moment tensors $\boldsymbol{E}^{s}(t), \boldsymbol{B}^{s}(t)$, etc are in general functions of time and set by the swimming gait.

This moments expansion allows us the flexibility to model particular swimmers or the interactions among many swimmers. For example, the spherical squirmers of Blake 28] and Ishikawa et al[29] invoke a quadrupolar moment $\boldsymbol{B}^{s}$ to achieve self-propulsion. The use of Stokesian dynamics to simulate various classes of 
microswimmers is given in Swan et al [30]. The only requirement for the slip velocity $\boldsymbol{u}^{s}$ is that it contributes no net translation or rotation to the particle (i.e., it has zero mean and zero antisymmetric first moment).

The total hydrodynamic force/torque on the swimmer can be written as

$$
\begin{aligned}
\mathcal{F}^{H} & =-\boldsymbol{R}_{\mathcal{F U}} \cdot \mathcal{U}-\underbrace{\boldsymbol{R}_{\mathcal{F E}}: \boldsymbol{E}^{s}-\boldsymbol{R}_{\mathcal{F B}} \odot \boldsymbol{B}^{s}-\cdots}_{\mathcal{F}^{\text {swim }}} \\
& =\mathcal{F}^{\text {drag }}+{ }_{t}
\end{aligned}
$$

where we grouped the force/torque $\mathcal{F}=\left(\boldsymbol{F}^{H}, \boldsymbol{L}^{H}\right)$ and translational/angular velocities $\mathcal{U}=(\boldsymbol{U}, \boldsymbol{\Omega})$, and the hydrodynamic resistance tensors $\boldsymbol{R}_{\mathcal{F U}}, \boldsymbol{R}_{\mathcal{F E}}, \boldsymbol{R}_{\mathcal{F B}}$, etc couple the force to the velocity and to the 'squirming set' $\boldsymbol{E}^{s}(t), \boldsymbol{B}^{s}(t)$, etc.

Although we motivated this discussion using a ciliated microorganism, the same development applies for self-diffusiophoretic Janus particles which also exhibit a fluid 'slip velocity' near the particle surface due to solvent backflow induced by the flux of chemical reactants/products along the particle surface. In fact, we can generalize this structure to all classes of microswimmers by recognizing that the resistance tensors are now functions of time, rather than being fixed for the ciliates.

Equation 1 has been written as a sum of the hydrodynamic drag force $\mathcal{F}^{\text {drag }}$ and self-propulsive 'swim force' $\mathcal{F}^{\text {swim }}$. A microswimmer moves in the Stokes regime, so its motion is 'force-free': $\sum \mathcal{F}=\mathcal{F}^{H}+\mathcal{F}^{e x t}=$ 0 , where $\mathcal{F}^{e x t}$ is any external force such as gravity. In the absence of an external field, $\mathcal{F}^{e x t}=0$ and we have $\mathcal{F}^{H}=0$. Using Eq1, we obtain $\mathcal{F}^{H}=\mathcal{F}^{\text {drag }}+\mathcal{F}^{\text {swim }}=0$ and thus the velocity of the swimmers is $\mathcal{U}=\boldsymbol{R}_{\mathcal{F U}}^{-1} \cdot \mathcal{F}^{\text {swim }}$.

For the simplest model of self-propelling spheres, the hydrodynamic resistance tensor is the Stokes drag factor $\boldsymbol{R}_{F U}=\zeta \boldsymbol{I}$, where $\zeta=6 \pi \eta a, a$ is the particle size and $\eta$ is the viscosity of the suspending Newtonian fluid; the swim force is $\boldsymbol{F}^{\text {swim }}=\zeta U_{0} \boldsymbol{q}$, where $U_{0}$ is the intrinsic swimming speed of the swimmer and $\boldsymbol{q}$ is a unit orientation vector prescribing the direction of swimming. The orientation vector $\boldsymbol{q}$ fluctuates subject to run-and-tumble or rotational Brownian diffusion and follows directly from a torque balance. In the absence of external forces, the translational velocity of the swimmer is therefore $\boldsymbol{U}=\boldsymbol{R}_{F U}^{-1} \cdot \boldsymbol{F}^{s w i m}=U_{0} \boldsymbol{q}$.

Equation 1 is the definition of the 'swim force' - one way to interpret this quantity is to measure the force required to prevent an active swimmer from moving, say by optical tweezers. In this case, the optical tweezer exerts an external force $\boldsymbol{F}^{\text {ext }}$ that exactly balances $\boldsymbol{F}^{\text {swim }}$ such that $\boldsymbol{F}^{\text {drag }}=\mathbf{0}$. The magnitude of the force required to hold the swimmer fixed is precisely $\left|\boldsymbol{F}^{\text {ext }}\right|=\left|\boldsymbol{F}^{\text {swim }}\right|$.

Including the effects of translational Brownian forces $\left(\boldsymbol{F}^{B}\right)$, external forces $\left(\boldsymbol{F}^{e x t}\right)$, and interparticle interactions $\left(\boldsymbol{F}^{P}\right)$ between the particles, this simple system is called the 'active Brownian particle' (ABP) model, where the force balance is $\mathbf{0}=-\zeta \boldsymbol{U}+\boldsymbol{F}^{\text {swim }}+\boldsymbol{F}^{B}+\boldsymbol{F}^{e x t}+\boldsymbol{F}^{P}$. Because the Brownian force can be $\mathcal{O}\left(10^{3}\right)$ times (or more) smaller than the self-propulsive swim force, $\boldsymbol{F}^{B}$ is often assumed to be negligible.

With an understanding of the self-propulsive forces that govern the motion of active systems, in the next section we turn our attention to the dynamic motion exhibited by active particles. 


\section{Diffusion: rotation leads to translation}

Suppose we have a self-propelling swimmer of characteristic size $a$ immersed in a continuous Newtonian solvent with viscosity $\eta$. The swimmer translates with a constant, intrinsic swim speed $U_{0}$ and tumbles with a reorientation time $\tau_{R}$. The reorientation time may be from run-and-tumble motion with $\tau_{R} \sim 1 / \omega$ where $\omega$ is the tumbling frequency and/or from the rotational Brownian motion with $\tau_{R} \sim 1 / D_{R} \sim 8 \pi \eta a^{3} /\left(k_{B} T\right)$ - there is an equivalence between reorientations induced by run-and-tumble and rotational Brownian motion 31 . For times large compared to $\tau_{R}$ (i.e., swimmer has undergone many reorientation events), the swimmer's trajectory can be modeled as a random-walk process.

The diffusivity for a random-walk scales as $D \sim l^{2} / \tau_{R}$ where $l$ is the step size. For active swimmers, the step size is the swimmer's run length $l=U_{0} \tau_{R}$ (or persistence length), which is simply the distance traveled between reorientation events. Therefore, the 'swim diffusivity' of the active body due to its self-motion scales as $D^{\text {swim }} \sim U_{0}^{2} \tau_{R}$. A rigorous theoretical analysis gives $D^{\text {swim }}=U_{0}^{2} \tau_{R} / 6$ in $3 \mathrm{D}$ and $D^{\text {swim }}=U_{0}^{2} \tau_{R} / 2$ in 2D for ABPs and similarly for run-and-tumble particles 22. With the effect of translational Brownian motion, the effective translational diffusivity $D^{e f f}=D_{0}+U_{0}^{2} \tau_{R} / 6$ where $D_{0}=k_{B} T / \zeta$ is the Stokes-EinsteinSutherland translational diffusivity. The swim diffusivity $D^{\text {swim }}$ can be more than $\mathcal{O}\left(10^{3}\right)$ larger than $D_{0}$.

Suppose we confine this swimmer with a container made of walls permeable to the fluid but not to the swimmer (i.e., an osmotic barrier). Because of the swimmer's tendency to wander away in space given by $D^{\text {swim }}$, it will exert a force or a pressure on the surrounding boundaries of the box as it collides into the walls. This pressure exerted on the surrounding walls to confine the particle is precisely the physical origin of the 'swim pressure' [5]. The swim pressure is conceptually similar to the kinetic theory of gases, where molecular collisions with the container walls exert a pressure, or to the Brownian osmotic pressure exerted by molecular or colloidal solutes in solution. It is therefore an entropic quantity that is driven by the constituent's tendency to diffuse, to undergo a random-walk. Although it is clear that such a swim pressure should exist, how are we to understand this pressure in basic physical quantities?

\section{Swim pressure of active matter}

The virial theorem expresses the stress $\boldsymbol{\sigma}$ (or pressure) on a system in terms of the forces $\boldsymbol{F}_{i}$ acting on it: $\boldsymbol{\sigma}=-1 / V\left\langle\sum_{i}^{N} \boldsymbol{x}_{i} \boldsymbol{F}_{i}\right\rangle$, where $\boldsymbol{x}_{i}$ is the position of particle $i, V$ is the system volume, and $N$ is the total number of particles 32. Suppose we have a particle in Stokes flow obeying the overdamped equation of motion, $\mathbf{0}=-\zeta \boldsymbol{U}(t)+\boldsymbol{F}(t)$, where $\zeta$ is the hydrodynamic drag factor, $\boldsymbol{U}$ is the particle velocity, and $\boldsymbol{F}$ is any general force on the body. The position of the particle at time $t$ is $\boldsymbol{x}(t)=\int \boldsymbol{U}\left(t^{\prime}\right) d t^{\prime}$, so we obtain the stress on the particle $\boldsymbol{\sigma}=-n\langle\boldsymbol{x} \boldsymbol{F}\rangle=-n \zeta \int\left\langle\boldsymbol{U}\left(t^{\prime}\right) \boldsymbol{U}(t)\right\rangle d t^{\prime}=-n \zeta \boldsymbol{D}$, where $n=N / V$ is the number density and we have written the time integral of the velocity autocorrelation as the diffusivity of the particle, $\boldsymbol{D}$.

This result demonstrates that a particle undergoing any type of random motion exerts a pressure $\Pi=$ $-\operatorname{tr} \sigma / 3=n \zeta D$. This general result applies for an arbitrary particle shape (where $\zeta$ may depend on particle 
configuration) and for any source of random motion. For passive Brownian particles where the source of random motion is the thermal energy, $D=k_{B} T / \zeta$, we obtain the familiar ideal-gas Brownian osmotic pressure $\Pi^{B}=n k_{B} T$. The osmotic pressure can be interpreted as a mechanical pressure resulting from the random motion induced by solvent fluctuations.

Likewise, for active particles with diffusivity $D^{\text {swim }}=U_{0}^{2} \tau_{R} / 6$, we arrive at the analogous "ideal-gas" swim pressure:

$$
\begin{aligned}
\Pi^{\text {swim }}(\phi \rightarrow 0) & =n \underbrace{\left(\zeta U_{0}^{2} \tau_{R} / 6\right)} \\
& =n\left(k_{s} T_{s}\right),
\end{aligned}
$$

where $\phi=4 \pi a^{3} n / 3$ is the volume fraction of active particles. As expected for dilute systems, $\Pi^{\text {swim }}$ depends on the particle size only through the hydrodynamic drag factor $\zeta$. In Eq 2 we have made an analogy to the Brownian osmotic pressure $\Pi^{B}=n k_{B} T$ and defined the 'activity' of the swimmers $k_{s} T_{s} \equiv \zeta U_{0}^{2} \tau_{R} / 6$. Because the entropic nature of $D^{\text {swim }}$ (and by extension $\Pi^{\text {swim }}=n \zeta D^{\text {swim }}$ ) comes not from the thermal energy but instead from swimmer self-propulsion and reorientation, the swim pressure is entirely athermal in origin. In two dimensions, $\Pi^{s w i m}=n \zeta U_{0}^{2} \tau_{R} / 2$. To appreciate the magnitude of this swimmer activity, a $1 \mu \mathrm{m}$ swimmer traveling in water with speed $U_{0} \sim 10 \mu \mathrm{m} / \mathrm{s}$ and reorienting in time $\tau_{R} \sim 10 \mathrm{~s}$ has an activity $k_{s} T_{s} \equiv \zeta U_{0}^{2} \tau_{R} / 6 \approx 4 p N \cdot \mu m$. The thermal energy at room temperature is $k_{B} T \approx 4 \times 10^{-3} p N \cdot \mu m$, meaning that the swimmers' intrinsic self-propulsion is equivalent to approximately $1000 k_{B} T$. In practice the intrinsic activity of active synthetic colloidal particles and living microorganisms can be even larger.

Returning to the virial theorem, we can take the forces $\boldsymbol{F}_{i}$ to be the swim force $\boldsymbol{F}^{\text {swim }}$ as discussed earlier and define the swim stress as

$$
\boldsymbol{\sigma}^{\text {swim }}=-n\left\langle\boldsymbol{x} \boldsymbol{F}^{\text {swim }}\right\rangle,
$$

and the swim pressure is the trace of the swim stress, $\Pi^{\text {swim }}=-\operatorname{tr} \boldsymbol{\sigma}^{\text {swim }} / 3$ in 3D. Equation 3 defines the swim stress as the first moment of the self-propulsive swim force $\boldsymbol{F}^{\text {swim }} \sim \zeta \boldsymbol{U}_{0}$, and the "moment arm" is the run length of the swimmer, $\boldsymbol{x} \sim \boldsymbol{U}_{0} \tau_{R}$. Equation 3 demonstrates the importance of interpreting the self-propulsion of an active particle as arising from a swim force, $\boldsymbol{F}^{\text {swim }}$. Unlike the familiar $-\left\langle\boldsymbol{x}_{i j} \boldsymbol{F}_{i j}\right\rangle$ form seen in interparticle interactions of molecular liquids, where subscripts $i j$ indicate pairwise interactions, $-\left\langle\boldsymbol{x} \boldsymbol{F}^{\text {swim }}\right\rangle$ gives a single-particle self contribution to the stress - just like the Brownian osmotic pressure $\Pi^{B}=n k_{B} T$.

This swim pressure exists at all scales in both living (e.g., microorganisms) and nonliving active systems, and also applies to larger swimmers (e.g., fish) where inertia is important (i.e., the Reynolds number is not small). A recent study on sedimentation [33] provided an indirect measurement of the swim pressure based upon density profiles of active particles under gravity. Acoustic traps may be used to expose an active system to a near-harmonic potential well, and the restricted swimmer motion inside the trap gives directly the swim pressure as defined via the virial theorem 34 . 
The swim pressure is distinct from the "hydrodynamic stresslet" that accompanies microswimmers, 35, 29] which scales as $n \zeta U_{0} a(\boldsymbol{q q}-\boldsymbol{I} / 3)$ and averages to zero for an isotropic distribution, where $a$ is the characteristic size of the swimmer.

The swim pressure of active matter is a real, measurable mechanical pressure exerted on a confining container. Suppose we load a soft, compressible material (e.g., gel polymer network) with photo-activated synthetic colloidal particles. In the absence of light, the particles undergo thermal Brownian motion and the gel assumes an equilibrium shape, determined by a balance between the entropic force that drives the polymer to expand and the elastic force that resists expansion [36. When the light is turned on, the particles suddenly become active and exert the swim pressure (Eq 2), causing the gel to expand isotropically. To make an appreciable change to the gel shape, the magnitude of the swim pressure must be larger than the shear modulus of the polymer network, which in principle an be adjusted to nearly zero. For example, a dilute network of hydrated mucus (a non-Newtonian gel) has shear moduli $\sim \mathcal{O}(0.1-10) P a$ 37, 38. The swim pressure exerted at $10 \%$ volume fraction of $1 \mu \mathrm{m}$ active particles in water with $U_{0} \sim 10 \mu \mathrm{m} / \mathrm{s}$ and $\tau_{R} \sim 10 \mathrm{~s}$ is $\Pi^{\text {swim }}=n \zeta U_{0}^{2} \tau_{R} / 6 \approx \mathcal{O}(1) P a$. For soft materials with a very small shear modulus, the swim pressure can cause the gel to deform its shape. Even if the gel does not deform, it can still be translated and be steered using the active swimmers [39. This suggests an application of active soft materials as micro- or nanomechanical devices that could have multiple applications in medicine (e.g., focused drug delivery), biophysics, and other fields. Others have analyzed the swim pressure in confinement between parallel plates [40] and along other geometric contours 41].

In addition to its practical applications, the swim pressure may be the basic underlying physical mechanism responsible for self-assembly and pattern formation in all active matter, as discussed next.

\section{Collective behavior of active matter}

An early numerical work by Vicsek et al[42] showed that a minimal kinetic model for active systems may result in their directed, coherent motion, illustrating that self-motion with some nominal interaction alone is enough to observe novel forms of phase behavior. Aditi Simha and Ramaswamy 43 and Saintillan and Shelley [35] developed a kinetic model with hydrodynamic interactions to predict the instabilities and pattern formation in rodlike active suspensions. More recently, experiments and computer simulations [19, 31, 44, 45, 46, 47, 48, have shown that active matter self-organizes into dense and dilute phases resembling an equilibrium liquid-gas coexistence. A phase separation in a classical thermodynamic system in equilibrium may occur due to attractive interactions between the molecules. Remarkably, for active matter these collective effects can occur in the absence of any attractive forces between the particles. How can purely excluded-volume or repulsive interactions give rise to attraction?

Continuum descriptions [48, 49] and micromechanical approaches such as structure factor analysis have provided models for this peculiar behavior [46, 47, 49, 50, 51, 52]. Tailleur, Cates, and coworkers [31, 45, 53] have developed a robust theory to explain the motility-induced phase separation in active matter using a 
flux-based Smoluchowski analysis. They developed an accurate continuum theory by explicit coarse graining and deriving the first-order density gradient expressions for a phase-separating active system with repulsive interactions [48]. This approach was further developed by analyzing the role of dimensionality [50], where the authors invoked the first moment of the static structure factor to predict the onset of instability. By considering a density-dependent particle swim velocity, they demonstrated that an effective chemical potential and a bulk free energy can be used to establish a mapping between particle-based active Brownian simulations and their continuum model. A generic mechanism for pattern formation and instability for reproducing and interacting run-and-tumble bacteria was also presented [45], by incorporating a varying local swim speed owing to different bacterial behavior in different environments.

Redner et al[46] analyzed the structural changes associated with phase separation and developed a simple kinetic model to predict the onset of instability, which was subsequently used to analyze a mixture of active and passive particles [54. The effect of interparticle collisions between the active particles was considered by Bialké et al[47] to derive a density-dependent effective particle swim speed. They further developed their nonlinear microrhology approach to predict the phase separation of self-propelled 2D disks [55].

Recently, an alternative approach invoking the unique mechanical pressure exerted by self-propelled bodies has been used to predict the self-assembly in active matter [5, 6, 56, 57]. The swim pressure [5] perspective offers a convenient framework to understand the collective behavior in active systems. Below, we summarize the use of the swim pressure to predict the phase separation in a system of active Brownian particles with a homogeneous activity, and to demonstrate that this simple system engenders a pressurevolume phase diagram much like that of a van der Waals fluid.

Physically, the swim pressure is the mechanical force per unit area that a confined active particle exerts on its container, given by Eq1 for a dilute active system. At higher concentrations of swimmers, the particles collide into each other and the swimmer size $a$ enters as a new variable in the problem. The nondimensional reorientation "Péclet number" $P e_{R}=U_{0} a / D^{\text {swim }}=U_{0} a /\left(U_{0}^{2} \tau_{R}\right)=a /\left(U_{0} \tau_{R}\right)$ is the ratio of the swimmer size $a$ to its run length $U_{0} \tau_{R}$, and this is a key parameter that determines the behavior of the swim pressure at higher swimmer concentrations, and the overall phase behavior of the system.

\subsection{Density dependence of swim pressure}

For large $P e_{R}$ the swimmers reorient rapidly and take small swim steps, behaving as if they are passive (inactive) particles subject to thermal Brownian motion with an effective activity $k_{s} T_{s} \equiv \zeta U_{0}^{2} \tau_{R} / 6[5]$. As shown in Fig 2A, our Brownian dynamics simulations for $P e_{R} \gg 1$ show that the swim pressure increases linearly with concentration. This system is analogous to passive Brownian particles, which exert the "ideal-gas" Brownian osmotic pressure $\Pi^{B}=n k_{B} T$ regardless of the concentration of particles. Thus $\Pi^{\text {swim }}\left(\phi, P e_{R}\right)=n k_{s} T_{s}$ as $P e_{R} \rightarrow \infty$ for all $\phi \lesssim \phi_{0}$ where $\phi_{0}$ is the volume fraction at close packing. Near

close packing the swimmers collide into each other before being allowed to take a swim step, so that swim pressure decreases to zero. 
For small $P e_{R}$ the swimmers have run lengths large compared to their size and hinder each others' movement during collisions. Suppose we have a cluster of particles with zero net cluster velocity, i.e., individual swim velocities cancel out due to collisions. Because the cluster does not move, the constituent swimmers have no effective run length and exert zero force on the surrounding walls of the container. This continues for a time $\tau_{R}$ until their swimming directions change from rotational motion and the cluster breaks apart. This "clustering" behavior 31] reduces the average distance each swimmer travels between reorientations, which decreases the pressure they exert on the container walls (i.e., the swim pressure). Another interpretation is that an active swimmer in larger concentrations is less mobile and has a smaller swim diffusivity, resulting in a smaller pressure via $\Pi^{\text {swim }}=n \zeta D^{\text {swim }}$. Therefore, for small $P e_{R}$ the swim pressure decreases as the swimmer concentration increases as shown in Fig 2 A. This differentiates active matter from an equilibrium Brownian system, which exerts a fixed $\Pi^{B}=n k_{B} T$ of ideal-gas pressure for all $\phi$.

Extending the results of a nonlinear microrheology analysis [5] the swim pressure at small $P e_{R}$ in $3 \mathrm{D}$ takes the form $\Pi^{\text {swim }}=n k_{s} T_{s}\left(1-\phi-\phi^{2}\right)[56$. Unlike Brownian systems where repulsive interactions (e.g., hardsphere collisions) increase the pressure, for active matter interactions decrease the run length and therefore the swim pressure. A decreasing $\Pi^{\text {swim }}$ is the principle destabilizing term that facilitates a phase transition in active systems.

\subsection{Interparticle (collisional) pressure}

At higher concentrations of active swimmers there is an additional contribution to the pressure due to interparticle (e.g., excluded volume) forces between the particles. Like the swim pressure and the Brownian osmotic pressure, the interparticle pressure is defined by the virial theorem: $\Pi^{P}=n\left\langle\boldsymbol{x} \cdot \boldsymbol{F}^{P}\right\rangle / 3$, where $\boldsymbol{F}^{P}$ is the interparticle force. As shown in Fig $2 \mathrm{~B}, \Pi^{P}$ necessarily increases with increasing concentration because excluded-volume collisions always result in a positive interparticle pressure, helping to stabilize the system.

For large $P e_{R}$ the swimmers behave as Brownian particles and $\Pi^{P}\left(\phi, P e_{R}\right)=\Pi^{H S}(\phi)$, where $\Pi^{H S}(\phi)$ is the interparticle pressure of hard-sphere Brownian particles [58, [59]. Because the detailed interactions between the particles are not important [58, 59, 60, the interparticle pressure for a molecular fluid or that of a Brownian colloidal system has the same density dependence as that of active swimmers. For large $P e_{R}$ the run length $U_{0} \tau_{R}$ sets the scale of the force moment and $\Pi^{P} \sim n\left(n a^{3}\right)\left(\zeta U_{0}\right)\left(U_{0} \tau_{R}\right) \sim n k_{s} T_{s} \phi$, analogous to the passive hard-sphere Brownian collisional pressure $\sim n k_{B} T \phi$.

For small $P e_{R}, \Pi^{P} \sim n\left(n a^{3}\right)\left(\zeta U_{0}\right) a$ because a swimmer is displaced by its size $a$ upon collision, not the run length $U_{0} \tau_{R}$, and the particle size $a$ sets the scale for the force moment. The interparticle pressure for small $P e_{R}$ in 3D can be modeled as $\Pi^{P}=3 n k_{s} T_{s} \phi P e_{R} g(2 ; \phi)$ [56], where $g(2 ; \phi)$ is the pair-distribution function at contact [59]. This scaling is different from $\Pi^{\text {swim }} \sim n\left(\zeta U_{0}\right)\left(U_{0} \tau_{R}\right)$, which is a single-particle contribution and the run length $U_{0} \tau_{R}$ sets the scale for the force moment. The ratio of the interparticle pressure to the swim pressure is $\Pi^{P} / \Pi^{\text {swim }} \sim \phi P e_{R}$, which provides an additional interpretation of the reorientation Péclet number as a balance of the two individual pressure contributions. 


\subsection{Active pressure}

The total pressure of active matter (in the absence of hydrodynamic interactions) is given by $P=$ $p_{f}+\Pi^{a c t}$, where $\Pi^{a c t}=\Pi^{\text {swim }}+\Pi^{P}$ is the 'active pressure' and $p_{f}$ is the solvent pressure (which is arbitrary for an incompressible fluid and is set to zero). Comparing Figs $2 \mathrm{~A}$ and $2 \mathrm{~B}$, we have a competing contribution to the active pressure. Namely, as we increase swimmer concentration, $\Pi^{\text {swim }}$ decreases (destabilizing) whereas $\Pi^{P}$ increases (stabilizing). This competition may result in what would be a negative 'second virial coefficient' $B_{2}$, which implies two-body attractions and the possibility of a 'gas-liquid phase transition.' Attractions may give rise to a non-monotonic variation of pressure with concentration, known as a "van der Waals loop."

Remarkably, this serves as an answer to why self-assembling active systems exhibit an effective attraction despite having purely repulsive particle interactions. The clustering behavior of self-propelled particles reduces the swim pressure they contribute to the system, which destabilizes the homogeneous phase into separate dense and dilute phases.

As shown in Fig 3 , at low $\phi$ all data collapse onto the ideal-gas swim pressure given by Eq 2. At high $P e_{R}$, the interparticle pressure dominates and the total pressure increases monotonically with $\phi$. Because the swimmers take small swim steps and reorient rapidly for $P e_{R} \gg 1$, the active pressure agrees well with the Carnahan-Starling equation of state for passive Brownian hard-spheres (see blue dashed curve in Fig 3). As $P e_{R}$ is reduced below $\sim 0.03$, we observe a non-monotonic pressure profile resembling a van der Waals loop. The decrease in $\Pi^{a c t}$ for $P e_{R} \ll 1$ is caused by the reduction in swim pressure due to the particles' tendency to form clusters, reducing the average distance they travel between reorientations. As $\phi$ approaches close packing, the swim pressure decrease to zero (see Fig $2 \mathrm{~A}$ ) but the active pressure necessarily increases because the interparticle (excluded volume) pressure diverges to infinity (Fig $2 \mathrm{~B}$ ). It is in this limit where experiments and computer simulations [19, 44, 46, 47, 48, 61] have observed the self-assembly of active systems into dense and dilute phases resembling an equilibrium liquid-gas coexistence.

When designing an experiment or computer simulation, the size of the container or simulation cell must be large compared to the run length of the swimmers, $U_{0} \tau_{R}$. A smaller container artificially reduces the swim pressure because the container size enters as a new length scale in the problem and diminishes the distance the swimmers travel between reorientations $34,40,62$.

We now understand the behavior of the active pressure for small and large values of $P e_{R}$; in the next subsection we discuss a simple model to predict the phase separation for all values of density and $P e_{R}$.

\subsection{PVT phase diagram}

Given an analytical expression for $\Pi^{\text {swim }}$ and $\Pi^{P}\left[56\right.$, the active pressure for small $P e_{R}$ is

$$
\Pi^{a c t}=n k_{s} T_{s}\left(1-\phi-\phi^{2}+3 \phi P e_{R}\left(1-\phi / \phi_{0}\right)^{-1}\right) .
$$

Equation 4 may be treated as an equation of state, which allows the prediction of phase separation in active matter. Recently Solon et al[63] evaluated the use and validity of the active pressure as a thermodynamic 
equation of state, arguing that the detailed interaction of an active particle with a solid wall may impact the pressure. However, we argue that the active pressure is a state function in general, which is explained in section 8 . For the purposes of our present discussion, we focus on spherical swimmers not in confinement, so there is no such wall interaction and we can use Eq 4 to predict the phase behavior.

Figure 4 shows the phase diagram in the $P e_{R}-\phi$ plane, where Eq 4 was used to determine the regions of stability from the spinodal condition, $\partial \Pi^{a c t} / \partial \phi=0$. This is given by the red curve in Fig 4 that passes through the extrema of each constant-pressure isocontour ("isobar"). At the critical point (red star in Fig 4, $\partial \Pi^{a c t} / \partial \phi=\partial^{2} \Pi^{a c t} / \partial \phi^{2}=0$. In $3 \mathrm{D}$ the critical volume fraction $\phi^{c} \approx 0.44$, active pressure $\Pi^{a c t, c} \phi^{c} /\left(n k_{s} T_{s}\right) \approx 0.21$, and reorientation Péclet number $P e_{R}^{c} \approx 0.028$, values consistent with our BD simulations and simulation data of others [64, 54, 55]. No notion of free energy is needed to obtain the spinodal and critical point - they are purely mechanical quantities.

The "binodal" or coexistence region (blue curve in Fig 4) is defined as the equality of the chemical potential in the dilute and dense phases. Although the thermodynamic chemical potential is defined only for equilibrium systems, we can define a nonequilibrium chemical potential for active systems using standard macroscopic mechanical balances [5, 56]: $n\left(\partial \mu^{a c t} / \partial n\right)=(1-\phi)\left(\partial \Pi^{a c t} / \partial n\right)$. This definition, which makes no approximation other than solvent incompressibility, agrees with the true thermodynamic chemical potential for molecular or colloidal solutes in solution[36]. Active systems with a small reorientation time $\tau_{R} \rightarrow 0$ not only behave similarly but are equivalent in dynamics to that of passive Brownian particles. If we placed active swimmers behaving identically to passive Brownian particles behind an osmotic barrier, we would not be able to distinguish one from the other. Because the form of the chemical potential and pressure are equivalent for the two systems, we interpret $\mu^{a c t}$ as a natural definition and extension of the chemical potential for nonequilibrium systems, and use it to compute and define a "binodal."

Simulations of Wysocki et al[64] agree well with the spinodal of this model as shown by the location of the transition from the homogeneous (open symbols) to phase-separated (filled symbols) states. In 2D, simulation of Speck et al[55] suggest that the transition occurs near the binodal [56]. Figure 4 which contains no adjustable parameters, predicts that active systems prepared outside the binodal (blue curve) are stable in the homogeneous configuration and do not phase separate. Between the spinodal and binodal, the system is in a metastable state and does not spontaneously undergo a spinodal decomposition. In a simulation the system may stay in the homogeneous phase unless an artificial nucleation seed causes the system to transition to the globally-stable phase [46.

From Fig 4 , we see that the reorientation Péclet number plays an important role in the phase behavior of active systems. In addition to a ratio of the particle size to its run length, we can use the swim activity $k_{s} T_{s} \equiv \zeta U_{0}^{2} \tau_{R} / 6$ to rewrite the reorientation Péclet number as $P e_{R} \equiv a /\left(U_{0} \tau_{R}\right)=\zeta U_{0} a /\left(6 k_{s} T_{s}\right)$, which is interpreted as a ratio of the interactive energy of the swimmer - force times distance, $\zeta U_{0} \times a-$ to the swim activity $k_{s} T_{s}$.

Figure 4 reveals that phase separation becomes possible for small $P e_{R}=\zeta U_{0} a /\left(6 k_{s} T_{s}\right)$, or high $T_{s}$. This 
is opposite to a classical thermodynamic system where phase transitions occur at low temperatures. Yet some systems like temperature-responsive polymers display a lower critical solution temperature (LCST) transition, where phase separation becomes possible at high temperatures [65]. A possible interpretation for active matter systems exhibiting a LCST is that the particle effectively becomes larger in size and thus has less space available for entropic mixing as $P e_{R}$ decreases (i.e., run length increases).

In the next section we discuss the notion of an effective 'temperature' of active matter in the context of phase separation and provide a justification that $T_{s}$ can indeed be interpreted as a temperature under certain situations.

\section{Temperature of active matter?}

Because the swim pressure has been a useful concept to predict the collective behavior of active matter, a natural question and extension pertains to the temperature of active matter. Wu and Libchaber [66] observed anomalous behavior of passive Brownian particles when placed in a suspension of run-and-tumble E. coli, and attributed the particles' enhanced translational diffusivity to the collective motion of the bacteria. Subsequent studies like Loi et al[67] introduced an interesting notion of using passive tracer particles as a 'thermometer' to measure the 'effective temperature' of an active suspension, as many experimental, numerical, and theoretical studies reported on the enhanced hydrodynamic tracer diffusion in a bacterial system $68,69,70,71,72,73,74,75$. However, the use of an effective temperature of nonequilibrium active matter is not valid in general[31, and it is unclear whether a mapping between the effective temperature and the thermodynamic temperature exists at all.

To understand the 'temperature' of active matter, we shall consider a simple experiment involving the mixing of two systems of active swimmers with a different activity, $k_{s} T_{s} \equiv \zeta U_{0}^{2} \tau_{R} / 6[76$. Suppose a system of "hot" active swimmers with $\left(k_{s} T_{s}\right)_{H}$ is initially separated from "cold" swimmers with $\left(k_{s} T_{s}\right)_{C}$. When the systems are allowed to mix, the swimmers with different activities collide and displace each other, but they never share their intrinsic kinetic activity $\left(k_{s} T_{s}\right)$ upon collisions. Even after a very long time, the system is still composed of "hot" and "cold" swimmers with no equilibration of the 'temperature,' or the activity. This is opposite to a purely passive Brownian suspension or a molecular fluid, which thermally equilibrates when systems of different temperatures mix.

For a passive tracer particle in a sea of active swimmers, the motion of the tracer depends on the swimmers' reorientation Péclet number $P e_{R} \equiv a /\left(U_{0} \tau_{R}\right)$. For $P e_{R} \gg 1$ the swimmers take small swim steps and they repeatedly displace the tracer particle of order the step size $\sim \mathcal{O}\left(U_{0} \tau_{R}\right)$ upon collisions. In this limit the tracer particle can sense the activity or 'temperature' of the swimmers via collisions because the fluctuations it receives come from the swimmers' activity (plus a contribution from the solvent's thermal fluctuations), allowing it to behave as a 'thermometer' for the activity $k_{s} T_{s}=\zeta U_{0}^{2} \tau_{R} / 6$. In this sense a suspension of swimmers with small run lengths $U_{0} \tau_{R}<a$ can be mapped to a purely Brownian suspension 
with an effective 'temperature' $k_{s} T_{s}^{1}$.

In the other limit of $P e_{R} \ll 1$, the swimmer collides with the tracer and continues to translate until the tracer moves completely clear of the swimmer's trajectory. The tracer receives a displacement of $\sim \mathcal{O}(a)$ upon colliding with a swimmer, not the run length $U_{0} \tau_{R}$. Unlike the limit of $P e_{R} \gg 1$, the tracer cannot act as a thermometer because it only receives a displacement of its size $a$, even though the swimmers actually diffuse with their swim diffusivity $D^{\text {swim }} \sim U_{0}^{2} \tau_{R}$. In this limit, the reorientation Péclet number $P e_{R} \equiv a /\left(U_{0} \tau_{R}\right)$ is the quantity that gets shared between the swimmers via collisions [56, and the activity $k_{s} T_{s} \equiv \zeta U_{0}^{2} \tau_{R} / 6$ cannot be mapped to the thermodynamic temperature.

Here we have focused on a simple system of active Brownian particles with a homogeneous intrinsic swim speed and reorientation time (i.e., activity), which lends itself to a thermodynamic perspective. There is also a continuum perspective which allows slow variations in space and time, and this engenders the notion of interpreting the swim force as a body force, as described next.

\section{Swim force as an 'internal' body force}

Recent discussions 63, 77, have questioned the validity of the active pressure as a true thermodynamic pressure and a state function. Solon et al 63 derived an expression for the pressure on the bounding walls of a container when a local, external torque was applied to each active particle colliding into the wall. They report that the wall pressure depends on the detailed form and nature of the local torque and conclude that the pressure of active matter thus cannot be a state function in general because the force per area on the bounding walls is not necessarily equal to the swim pressure far away from the wall, especially when polar order is present in the system.

A recent work resolved this concern by using both a global force balance and a continuum-level derivation[78]. We established in Sec 2 that it is permissible and essential to interpret the self-propulsion of an active particle as arising from a swim force, $\boldsymbol{F}^{\text {swim }}=\zeta U_{0} \boldsymbol{q}$ for an active Brownian particle, where $\boldsymbol{q}$ is the unit orientation vector specifying the particle's direction of swimming. For an active particle in the absence of any external forces or torques, the average swim force is zero because the particle's orientation distribution is uniform: $\left\langle\boldsymbol{F}^{\text {swim }}\right\rangle=\zeta U_{0}\langle\boldsymbol{q}\rangle=\mathbf{0}$. If we have an external orienting torque on the particles that result in polar order, then the swimming orientations are not uniform, $\langle\boldsymbol{q}\rangle \neq \mathbf{0}$, and thus there is a nonzero average swim force $\left\langle\boldsymbol{F}^{\text {swim }}\right\rangle \neq \mathbf{0}$. This nonzero average swim force may arise from an intrinsic mechanism internal to the body, so it is to be construed as an 'internal' force. Yet, this internal average swim force behaves equivalently to an external body force such as gravity 78 . Thus the true mechanical pressure exerted on a boundary is a sum of the swim pressure plus the 'weight' of the particles (or the average swim force), and the active pressure is indeed well-defined and independent of interactions with boundaries.

\footnotetext{
${ }^{1}$ For active Brownian particles, this contribution is in addition to the thermal $k_{B} T$ that gets shared as usual as a result of translational Brownian motion.
} 
A microscopic theory [62] based upon moment expansions of the Smoluchowski equation revealed that the particle concentration and hence the force exerted on the wall depends on boundary curvature, flux conditions at the surface, etc., which agree with Solon et al[63. Inclusion of the internal body force (i.e., nonzero average swim force) into the momentum balance shows that the force per unit area on the boundary plus the integral of the internal body force is equal to the active pressure far from the boundary.

The swim pressure perspective allows researchers to analyze the effects of external forces like gravity or orienting torques in a homogeneous suspension, and then use these results to subsequently predict the inhomogeneous behavior of the active system. External gravitational fields or torques do not cause the active particles to generate their own mechanical pressure, but the external fields can affect the swim pressure 39].

We motivated this development assuming that the particles have polar order and hence a nonzero $\left\langle\boldsymbol{F}^{\text {swim }}\right\rangle$. However, we may also have a density-dependent or spatially-varying intrinsic swim velocity $U_{0}(\boldsymbol{x})$ and reorientation time $\tau_{R}(\boldsymbol{x})$, due to a variation in fuel concentration, for example. This results in a nonzero $\left\langle\boldsymbol{F}^{\text {swim }}\right\rangle=-(1 / n) \boldsymbol{\sigma}^{\text {swim }} \cdot \nabla \log \left(U_{0} \tau_{R}\right)[76$, which must then appear in the global force balance and in the continuum description to compute the pressure of active matter. Therefore, the key is to have a nonzero $\left\langle\boldsymbol{F}^{\text {swim }}\right\rangle$, not necessarily any polar order.

In this Review we have assumed that the active particles do not change their swimming speed nor reorientation time upon increasing concentration. Both synthetic Janus swimmers and biological microorganisms may alter their activity based upon confinement or local swimmer concentration, and this would add an additional complexity and modify the swim pressure. However, the swim pressure would still be a fundamental concept to explain the phase-separating behavior of active systems.

Lastly, a microscopic perspective 62 allows any scale variation and shows the importance of another micro-length scale $\delta \sim \sqrt{D_{T} \tau_{R}}$ that allows the swim pressure to emerge naturally. This perspective allows the swimmers' run length to be on the order of the body size (or smaller) and gives rise to interesting phenomena like the Casimir effect[79].

\section{Conclusions}

In this Review we discussed the forces and stresses generated by active matter, and the (thermo)dynamics that results from the activity of self-propelled Brownian microswimmers. We focused on recent theoretical work predicting the fascinating collective behavior and phase separation in active systems. The nontrivial behavior of active matter such as spontaneous self-assembly and pattern formation [1, 2, 3] makes the understanding of their complex dynamics a challenging problem in the statistical physics of soft matter.

We discussed in detail about the notion that all active matter systems generate a swim pressure due to their self motion. The swim pressure is distinct from, and in addition to, the "hydrodynamic stresslet" 29, 35]. For larger swimmers where the Reynolds number is not small, we also have the Reynolds (or Bernoulli) pressure contribution $\rho\left\langle\boldsymbol{u}^{\prime} \cdot \boldsymbol{u}^{\prime}\right\rangle$, where $\rho$ is the density and $\boldsymbol{u}^{\prime}$ is the velocity fluctuation, in addition to the swim pressure. 
An exciting future development is to analyze the extent to which the ideas presented here are applicable to larger swimmers such as fish and birds, and whether the swim pressure perspective may be used to explain large-scale flocking behavior. Another important ongoing challenge in active soft matter has been to understand the influence of solvent-mediated hydrodynamic interactions on the behavior of active systems. The simple model presented in Sec6 6 neglected the effects of hydrodynamic interactions between the particles, which would contribute additional terms to the active pressure and affect the reorientation time. Including the effects of hydrodynamic interactions is an arduous task because the dynamics of the surrounding fluid must be incorporated into the model, in addition to that of the active particles.

On the experimental side, we foresee the use of swim pressure concepts in real-life engineering applications, such as the fabrication of novel soft materials using active swimmers. We also believe that the swim pressure may have forthcoming applications in molecular-cell biology, as the determination of the mechanical forces and stresses generated by active constituents inside a living cell may engender new discoveries in cellular morphology and function. Lastly, further experimental evidence for the intriguing behavior of active systems, such as the Casimir phenomenon or the effect of external polar or nematic fields on collective motion, is a forthcoming development in this area.

Active matter has engendered a new field of fundamental physics, materials science, and biology. Cellular biology and biophysics are becoming increasingly concerned with the mechanical forces, stresses, and (thermo)dynamics inside a living cell. New frameworks have been developed by many soft matter researchers around the world to make predictions and corroborate the fascinating nonequilibrium behaviors exhibited by active matter. Given the richness and challenges inherent in active soft matter, much work remains to elucidate the nonequilibrium dynamics of living systems and other far from equilibrium systems.

\section{Acknowledgments}

SCT is supported by a Gates Millennium Scholars fellowship and a National Science Foundation (NSF) Graduate Research Fellowship (No. DGE-1144469). This work is also supported by NSF Grant CBET 1437570 .

\section{References}

[1] D. J. Sumpter, Collective Animal Behavior, Princeton University Press, Princeton, New Jersey, USA, 2010.

[2] S. Ramaswamy, The mechanics and statistics of active matter, Ann Rev Condens Matter Phys 1 (1) (2010) 323-345.

** Of outstanding interest as this work reviews the nonequilibrium statistical mechanics and hydrodynamics of active systems displaying collective behavior.

[3] J. Toner, Y. Tu, S. Ramaswamy, Hydrodynamics and phases of flocks, Ann Phys 318 (1) (2005) 170-244. 
[4] M. C. Marchetti, J. F. Joanny, S. Ramaswamy, T. B. Liverpool, J. Prost, M. Rao, R. A. Simha, Hydrodynamics of soft active matter, Reviews of Modern Physics 85 (3) (2013) 1143-1189.

* Of special interest as this work gives a thorough and comprehensive review of the mechanical and statistical properties of living systems.

[5] S. C. Takatori, W. Yan, J. F. Brady, Swim pressure: stress generation in active matter, Phys Rev Lett 113 (2) (2014) 028103.

** Of outstanding work as this article introduces the swim pressure as the notion that all active matter systems exert a unique mechanical pressure owing to their self-motion.

[6] X. Yang, M. L. Manning, M. C. Marchetti, Aggregation and segregation of confined active particles, Soft Matter 10 (34) (2014) 6477-6484.

** Of outstanding interest as this work identified that confined active particles exert a mechanical pressure on the walls of the container due to their self-motion.

[7] E. M. Purcell, Life at low reynolds number, American Journal of Physics 45 (1) (1977) 3-11.

[8] G. Taylor, The action of waving cylindrical tails in propelling microscopic organisms, Proceedings of the Royal Society of London A: Mathematical, Physical and Engineering Sciences 211 (1105) (1952) $225-239$.

[9] S. Nakata, Y. Hayashima, Spontaneous dancing of a camphor scraping, Journal of the Chemical Society, Faraday Transactions 94 (24) (1998) 3655-3658.

[10] R. F. Ismagilov, A. Schwartz, N. Bowden, G. M. Whitesides, Autonomous movement and self-assembly, Angewandte Chemie International Edition 41 (4) (2002) 652-654.

[11] W. F. Paxton, K. C. Kistler, C. C. Olmeda, A. Sen, S. K. St. Angelo, Y. Cao, T. E. Mallouk, P. E. Lammert, V. H. Crespi, Catalytic nanomotors: autonomous movement of striped nanorods, J Am Chem Soc 126 (41) (2004) 13424-13431.

** Of outstanding interest as this was one of the first experimental manifestation of self-propelling active Brownian colloids.

[12] J. R. Howse, R. A. L. Jones, A. J. Ryan, T. Gough, R. Vafabakhsh, R. Golestanian, Self-motile colloidal particles: from directed propulsion to random walk, Phys Rev Lett 99 (4) (2007) 048102.

[13] W. F. Paxton, P. T. Baker, T. R. Kline, Y. Wang, T. E. Mallouk, A. Sen, Catalytically induced electrokinetics for motors and micropumps, J Am Chem Soc 128 (46) (2006) 14881-14888.

[14] A. Ajdari, L. Bocquet, Giant amplification of interfacially driven transport by hydrodynamic slip: Diffusio-osmosis and beyond, Phys Rev Lett 96 (18) (2006) 186102. 
[15] R. Golestanian, T. B. Liverpool, A. Ajdari, Designing phoretic micro- and nano-swimmers, New Journal of Physics 9 (5) (2007) 126.

[16] U. M. Córdova-Figueroa, J. F. Brady, Osmotic propulsion: The osmotic motor, Phys Rev Lett 100 (15) (2008) 158303.

[17] A. Brown, W. Poon, Ionic effects in self-propelled pt-coated janus swimmers, Soft Matter 10 (22) (2014) $4016-4027$.

[18] H.-R. Jiang, N. Yoshinaga, M. Sano, Active motion of a janus particle by self-thermophoresis in a defocused laser beam, Phys Rev Lett 105 (26) (2010) 268302.

[19] J. Palacci, S. Sacanna, A. P. Steinberg, D. J. Pine, P. M. Chaikin, Living crystals of light-activated colloidal surfers, Science 339 (6122) (2013) 936-940.

[20] G. Volpe, I. Buttinoni, D. Vogt, H.-J. K ummerer, C. Bechinger, Microswimmers in patterned environments, Soft Matter 7 (19) (2011) 8810-8815.

[21] L. Baraban, D. Makarov, O. G. Schmidt, G. Cuniberti, P. Leiderer, A. Erbe, Control over janus micromotors by the strength of a magnetic field, Nanoscale 5 (4) (2013) 1332-1336.

[22] H. Berg, Random Walks in Biology, Princeton University Press, Princeton, New Jersey, USA, 1993.

[23] J. Adler, Chemotaxis in bacteria, Science 153 (3737) (1966) 708-716.

[24] R. Blakemore, Magnetotactic bacteria, Science 190 (4212) (1975) 377-379.

[25] J. Armitage, K. Hellingwerf, Light-induced behavioral responses ('phototaxis') in prokaryotes, Photosynth Res 76 (1-3) (2003) 145-155.

[26] D. Hader, R. Hemmersbach, M. Lebert, Gravity and the Behavior of Unicellular Organisms, Cambridge University Press, Cambridge, United Kingdom, 2005.

[27] X. Garcia, S. Rafa i, P. Peyla, Light control of the flow of phototactic microswimmer suspensions, Phys Rev Lett 110 (13) (2013) 138106.

[28] J. R. Blake, A spherical envelope approach to ciliary propulsion, J Fluid Mech 46 (01) (1971) 199-208.

* Of special interest as this work introduces a moments expansion to express the slip velocity at the surface of a microswimmer.

[29] T. Ishikawa, M. P. Simmonds, T. J. Pedley, Hydrodynamic interaction of two swimming model microorganisms, J Fluid Mech 568 (2006) 119-160.

[30] J. W. Swan, J. F. Brady, R. S. Moore, C. 174, Modeling hydrodynamic self-propulsion with stokesian dynamics. or teaching stokesian dynamics to swim, Phys Fluids 23 (7) (2011) 071901-071919. 
[31] M. E. Cates, J. Tailleur, When are active brownian particles and run-and-tumble particles equivalent? consequences for motility-induced phase separation, Europhys Lett 101 (2) (2013) 20010.

[32] H. Goldstein, Classical Mechanics, 2nd Edition, Addison-Wesley, Reading, MA, 1990.

[33] F. Ginot, I. Theurkauff, D. Levis, C. Ybert, L. Bocquet, L. Berthier, C. Cottin-Bizonne, Nonequilibrium equation of state in suspensions of active colloids, Phys Rev X 5 (1) (2015) 011004.

[34] S. C. Takatori, R. De Dier, J. Vermant, J. F. Brady, Acoustic trapping of active matter (2016) in review.

[35] D. Saintillan, M. J. Shelley, Instabilities, pattern formation, and mixing in active suspensions, Phys Fluids 20 (12) (2008) 123304-123315.

* Of special interest as the authors use a Smoluchowski-based moments expansion to predict instabilities in active fluids.

[36] M. Doi, Soft Matter Physics, Oxford University Press, Oxford, United Kingdom, 2013.

** Of outstanding interest as this is a clearly-written and versatile soft matter physics text.

[37] D. S. Fudge, T. Winegard, R. H. Ewoldt, D. Beriault, L. Szewciw, G. H. McKinley, From ultra-soft slime to hard $\alpha$-keratins: The many lives of intermediate filaments, Integrative and comparative biology 49 (1) (2009) 32-39.

[38] S. K. Lai, Y.-Y. Wang, D. Wirtz, J. Hanes, Micro- and macrorheology of mucus, Adv Drug Deliv Rev 61 (2) (2009) 86-100.

[39] S. C. Takatori, J. F. Brady, Swim stress, motion, and deformation of active matter: effect of an external field, Soft Matter 10 (47) (2014) 9433-9445.

[40] B. Ezhilan, R. Alonso-Matilla, D. Saintillan, On the distribution and swim pressure of run-and-tumble particles in confinement, J Fluid Mech 781 (2015) R4.

[41] F. Smallenburg, H. Lwen, Swim pressure on walls with curves and corners, Phys Rev E 92 (3) (2015) 032304 .

[42] T. Vicsek, A. Czirók, E. Ben-Jacob, I. Cohen, O. Shochet, Novel type of phase transition in a system of self-driven particles, Phys Rev Lett 75 (6) (1995) 1226-1229.

** Of outstanding interest as this work laid the groundwork for the 'Vicsek model,' perhaps the most famous and widely-used microscopic theory for collective motion.

[43] R. Aditi Simha, S. Ramaswamy, Hydrodynamic fluctuations and instabilities in ordered suspensions of self-propelled particles, Phys Rev Lett 89 (5) (2002) 058101.

** Of outstanding interest as this work develops the hydrodynamic equations for active suspensions with spontaneous orientational order. 
[44] I. Theurkauff, C. Cottin-Bizonne, J. Palacci, C. Ybert, L. Bocquet, Dynamic clustering in active colloidal suspensions with chemical signaling, Phys Rev Lett 108 (26) (2012) 268303.

* Of special interest as this work was one of the early experimental evidences of self-assembly in synthetic active Janus particles.

[45] M. E. Cates, D. Marenduzzo, I. Pagonabarraga, J. Tailleur, Arrested phase separation in reproducing bacteria creates a generic route to pattern formation, Proc Natl Acad Sci U.S.A 107 (26) (2010) 1171511720 .

** Of outstanding interest as this study provides a generic mechanism for pattern formation and instability for reproducing and interacting run-and-tumble bacteria by invoking a varying local swim speed.

[46] G. S. Redner, M. F. Hagan, A. Baskaran, Structure and dynamics of a phase-separating active colloidal fluid, Phys Rev Lett 110 (5) (2013) 055701.

[47] J. Bialké, H. Löwen, T. Speck, Microscopic theory for the phase separation of self-propelled repulsive disks, Europhys Lett 103 (3) (2013) 30008.

[48] J. Stenhammar, A. Tiribocchi, R. J. Allen, D. Marenduzzo, M. E. Cates, Continuum theory of phase separation kinetics for active brownian particles, Phys Rev Lett 111 (14) (2013) 145702.

** Of outstanding interest as the authors develop an accurate continuum theory by explicit coarse graining and deriving the first-order density gradient expressions for a phase-separating active system.

[49] Y. Fily, M. C. Marchetti, Athermal phase separation of self-propelled particles with no alignment, Phys Rev Lett 108 (23) (2012) 235702.

[50] J. Stenhammar, D. Marenduzzo, R. J. Allen, M. E. Cates, Phase behaviour of active brownian particles: the role of dimensionality, Soft Matter 10 (10) (2014) 1489-1499.

[51] R. Ni, M. A. C. Stuart, M. Dijkstra, Pushing the glass transition towards random close packing using self-propelled hard spheres, Nat Commun 4 (2013) 2704.

[52] A. Baskaran, M. C. Marchetti, Enhanced diffusion and ordering of self-propelled rods, Phys Rev Lett $101(26)(2008) 268101$.

[53] J. Tailleur, M. E. Cates, Statistical mechanics of interacting run-and-tumble bacteria, Phys Rev Lett 100 (21) (2008) 218103.

** Of outstanding work as this article is the first to use a Smoluchowski-based analysis to predict motility-induced phase separation in active matter.

[54] J. Stenhammar, R. Wittkowski, D. Marenduzzo, M. E. Cates, Activity-induced phase separation and self-assembly in mixtures of active and passive particles, Phys Rev Lett 114 (1) (2015) 018301. 
[55] T. Speck, J. Bialké, A. M. Menzel, H. Löwen, Effective cahn-hilliard equation for the phase separation of active brownian particles, Phys Rev Lett 112 (21) (2014) 218304.

[56] S. C. Takatori, J. F. Brady, Towards a thermodynamics of active matter, Phys Rev E 91 (3) (2015) 032117.

* Of special interest as this article tests whether concepts from classical thermodynamics can be applied to nonequilibrium active matter.

[57] Y. Fily, S. Henkes, M. C. Marchetti, Freezing and phase separation of self-propelled disks, Soft Matter 10 (13) (2014) 2132-2140.

[58] D. McQuarrie, Statistical Mechanics, University Science Books, Mill Valley, California, USA, 2000.

[59] W. Russel, D. Saville, W. Schowalter, Colloidal Dispersions, Cambridge University Press, 1992.

[60] J. F. Brady, Brownian motion, hydrodynamics, and the osmotic pressure, J Chem Phys 98 (4) (1993) $3335-3341$.

[61] I. Buttinoni, J. Bialké, F. Kümmel, H. Löwen, C. Bechinger, T. Speck, Dynamical clustering and phase separation in suspensions of self-propelled colloidal particles, Phys Rev Lett 110 (23) (2013) 238301.

[62] W. Yan, J. F. Brady, The force on a boundary in active matter, J Fluid Mech 785 (2015) R1.

[63] A. P. Solon, Y. Fily, A. Baskaran, M. E. Cates, Y. Kafri, M. Kardar, J. Tailleur, Pressure is not a state function for generic active fluids, Nat Phys 11 (8) (2015) 673-678.

[64] A. Wysocki, R. G. Winkler, G. Gompper, Cooperative motion of active brownian spheres in threedimensional dense suspensions, Europhys Lett 105 (4) (2014) 48004.

[65] R. B. Griffiths, J. C. Wheeler, Critical points in multicomponent systems, Phys Rev A 2 (3) (1970) $1047-1064$.

[66] X.-L. Wu, A. Libchaber, Particle diffusion in a quasi-two-dimensional bacterial bath, Phys Rev Lett 84 (13) (2000) 3017-3020.

* Of special interest as this study experimetally analyzed the effect of bacterial activity on the translational diffusivity of passive tracer particles.

[67] D. Loi, S. Mossa, L. F. Cugliandolo, Effective temperature of active matter, Phys Rev E 77 (5) (2008) 051111.

[68] G. Mino, T. E. Mallouk, T. Darnige, M. Hoyos, J. Dauchet, J. Dunstan, R. Soto, Y. Wang, A. Rousselet, E. Clement, Enhanced diffusion due to active swimmers at a solid surface, Phys Rev Lett 106 (4) (2011) 048102 . 
[69] T. V. Kasyap, D. L. Koch, M. Wu, Hydrodynamic tracer diffusion in suspensions of swimming bacteria, Phys Fluids 26 (8) (2014) 081901.

[70] K. C. Leptos, J. S. Guasto, J. P. Gollub, A. I. Pesci, R. E. Goldstein, Dynamics of enhanced tracer diffusion in suspensions of swimming eukaryotic microorganisms, Phys Rev Lett 103 (19) (2009) 198103.

[71] H. Kurtuldu, J. S. Guasto, K. A. Johnson, J. P. Gollub, Enhancement of biomixing by swimming algal cells in two-dimensional films, Proc Natl Acad Sci U.S.A 108 (26) (2011) 10391-10395.

[72] J.-L. Thiffeault, S. Childress, Stirring by swimming bodies, Phys Lett A 374 (34) (2010) 3487-3490.

[73] M. J. Kim, K. S. Breuer, Enhanced diffusion due to motile bacteria, Phys Fluids 16 (9) (2004) L78-L81.

[74] A. Jepson, V. A. Martinez, J. Schwarz-Linek, A. Morozov, W. C. K. Poon, Enhanced diffusion of nonswimmers in a three-dimensional bath of motile bacteria, Phys Rev E 88 (4) (2013) 041002.

[75] A. E. Patteson, A. Gopinath, P. K. Purohit, P. E. Arratia, Particle diffusion in active fluids is nonmonotonic in size.

[76] S. C. Takatori, J. F. Brady, A theory for the phase behavior of mixtures of active particles, Soft Matter 11 (40) (2015) 7920-7931.

[77] A. P. Solon, J. Stenhammar, R. Wittkowski, M. Kardar, Y. Kafri, M. E. Cates, J. Tailleur, Pressure and phase equilibria in interacting active brownian spheres, Phys Rev Lett 114 (19) (2015) 198301.

[78] W. Yan, J. F. Brady, The swim force as a body force, Soft Matter 11 (31) (2015) 6235-6244.

[79] D. Ray, C. Reichhardt, C. J. O. Reichhardt, Casimir effect in active matter systems, Phys Rev E 90 (1) (2014) 013019. 


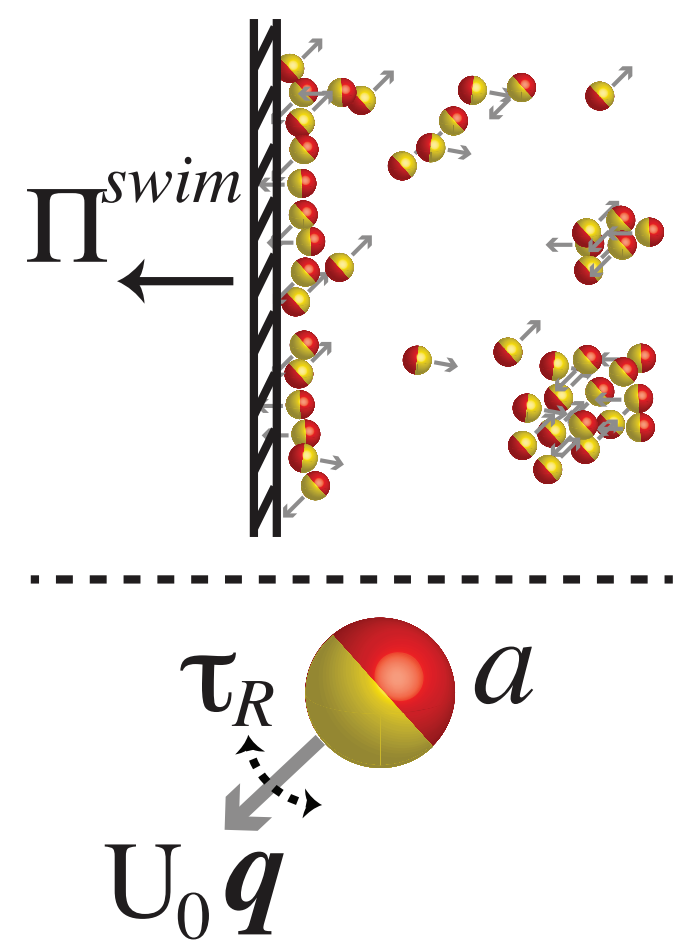

Figure 1: Self-propelled bodies exert a unique mechanical 'swim pressure' $\left[5\right.$, $\Pi^{\text {swim }}$, on an osmotic boundary owing to their self-motion. In a simple model of active matter, particles of size $a$ translate with a swim velocity $U_{0} \boldsymbol{q}$ and reorient with a reorientation time scale $\tau_{R}$, where the unit orientation vector $\boldsymbol{q}$ indicates the direction of swimming.
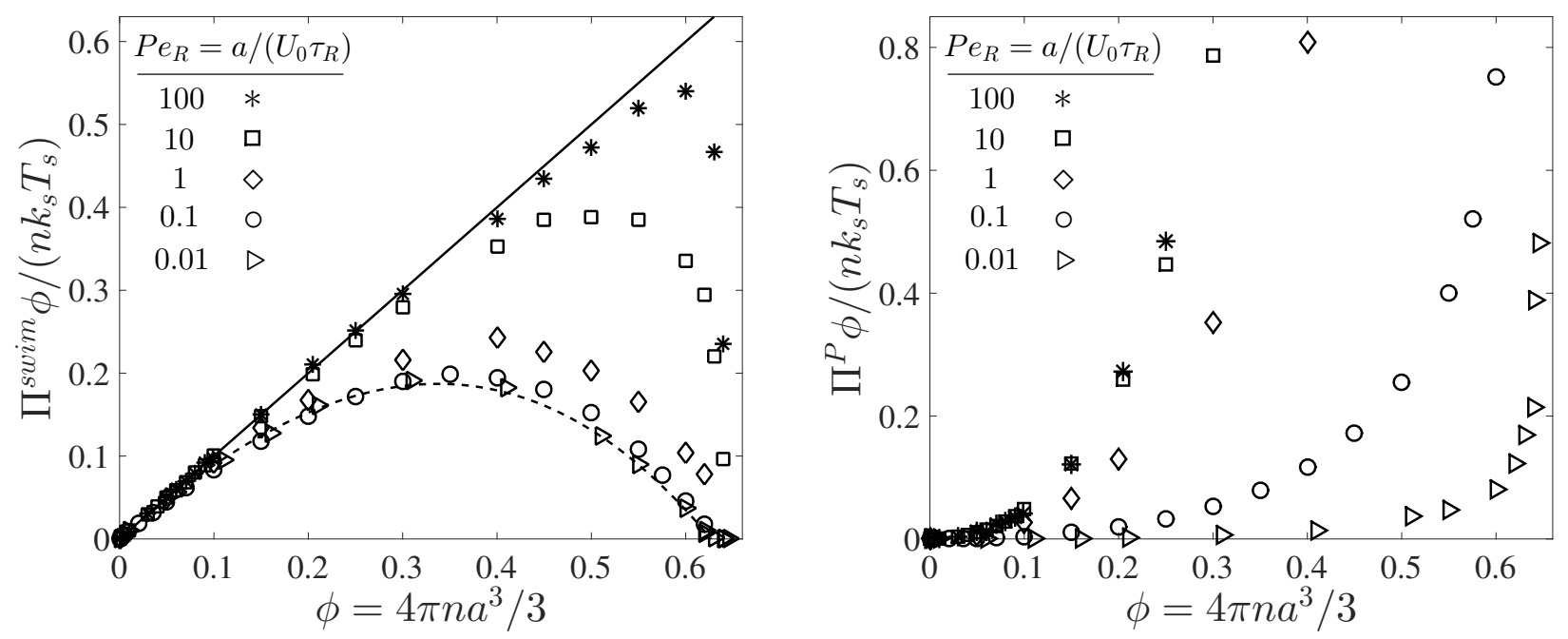

Figure 2: Dependence of swimmer concentration on (A) swim pressure and (B) interparticle (collisional) pressure scaled with the swim activity $k_{s} T_{s} \equiv \zeta U_{0}^{2} \tau_{R} / 6$. Data are from Brownian dynamics simulations, where the reorientation Péclet number $P e_{R} \equiv a /\left(U_{0} \tau_{R}\right)$ is the ratio of the swimmer size to its run length. In (A), for large $P e_{R}$ the data collapse on the solid line representing a linear increase of the active pressure with concentration, $\Pi^{s w i m}=n k_{s} T_{s}$. As $P e_{R} \rightarrow 0$ the swim pressure decreases with increasing concentration and agrees with $\Pi^{\text {swim }}=n k_{s} T_{s}\left(1-\phi-\phi^{2}\right)$ (dashed curve) [56]. In (B), the collisional pressure increases monotonically with concentration for all $P e_{R}$. 


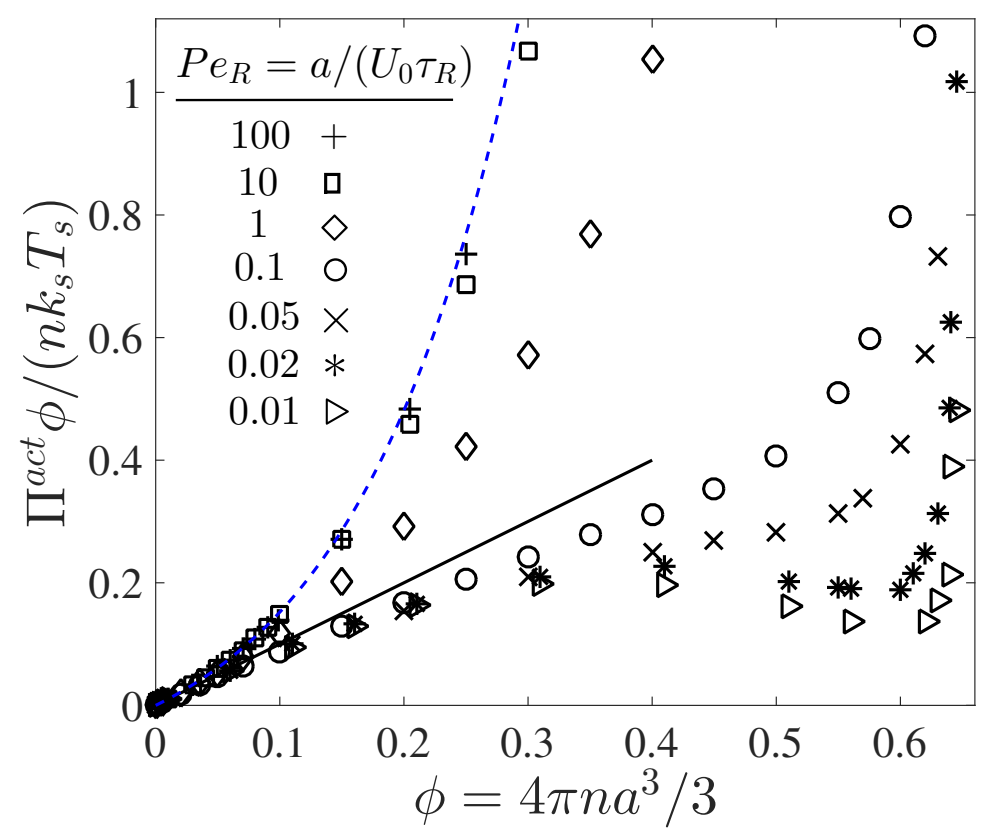

Figure 3: Nonequilibrium $\Pi^{a c t}-\phi$ phase diagram, where $\Pi^{a c t}=\Pi^{\text {swim }}+\Pi^{P}$ and is scaled with the swim activity $k_{s} T_{s} \equiv$ $\zeta U_{0}^{2} \tau_{R} / 6$. Data are from Brownian dynamics simulations, where the reorientation Péclet number $P e_{R} \equiv a /\left(U_{0} \tau_{R}\right)$ is the ratio of the swimmer size to its run length. The solid line represents a linear increase of the active pressure with concentration, $\Pi^{a c t}=n k_{s} T_{s}$. The dashed blue curve is the Carnahan-Starling equation of state for Brownian hard-spheres. For $P e_{R}<1 / 3$ we observe a negative 'second virial coefficient,' and for $P e_{R} \lesssim 0.03$ a non-monotonic pressure variation (analogous to a 'van der Waals loop'). Figure adapted from [5]. 


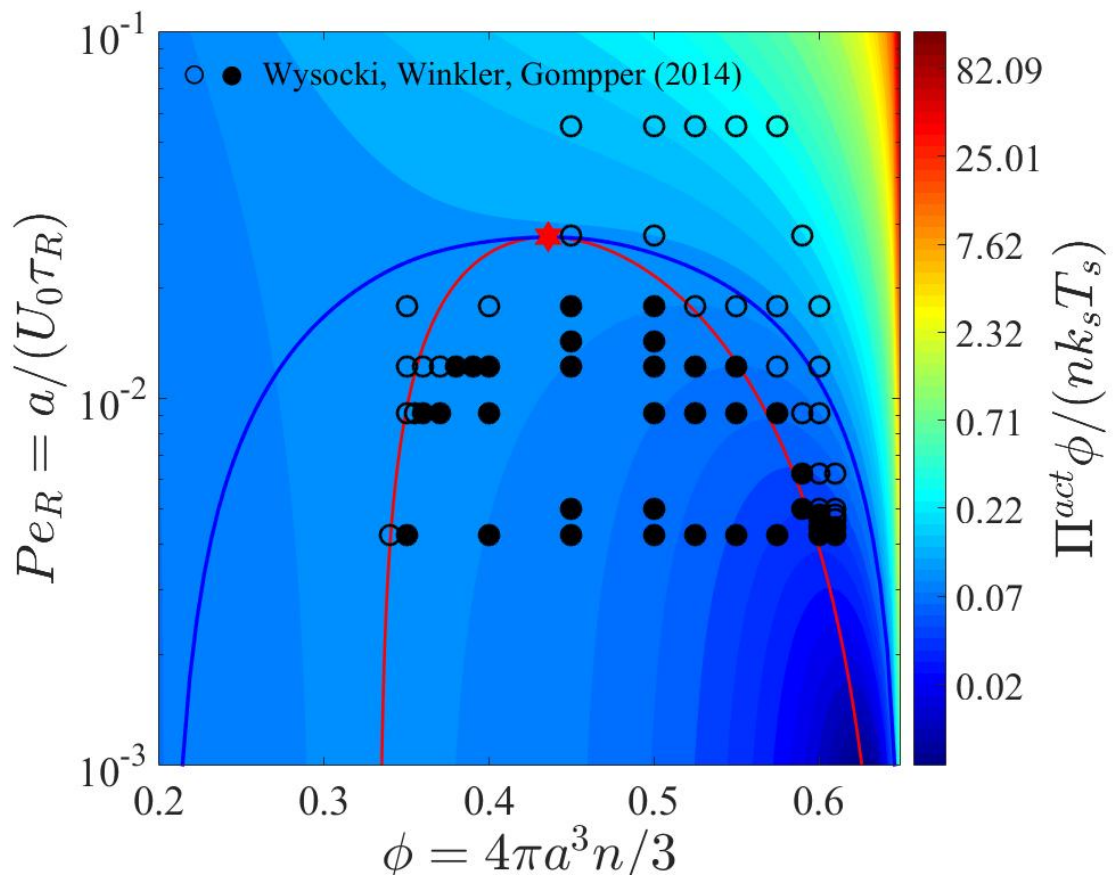

Figure 4: Phase diagram in the $P e_{R}-\phi$ plane for a 3D active system. Colorbar represents the magnitude of the active pressure scaled with the swim activity $k_{s} T_{s} \equiv \zeta U_{0}^{2} \tau_{R} / 6$, and the blue and red curves are the binodal and spinodal, respectively. The critical point is shown with a red star. The open and filled symbols are simulation data 64 with a homogeneous and phased-separated state, respectively. Figure adapted from [56]. 


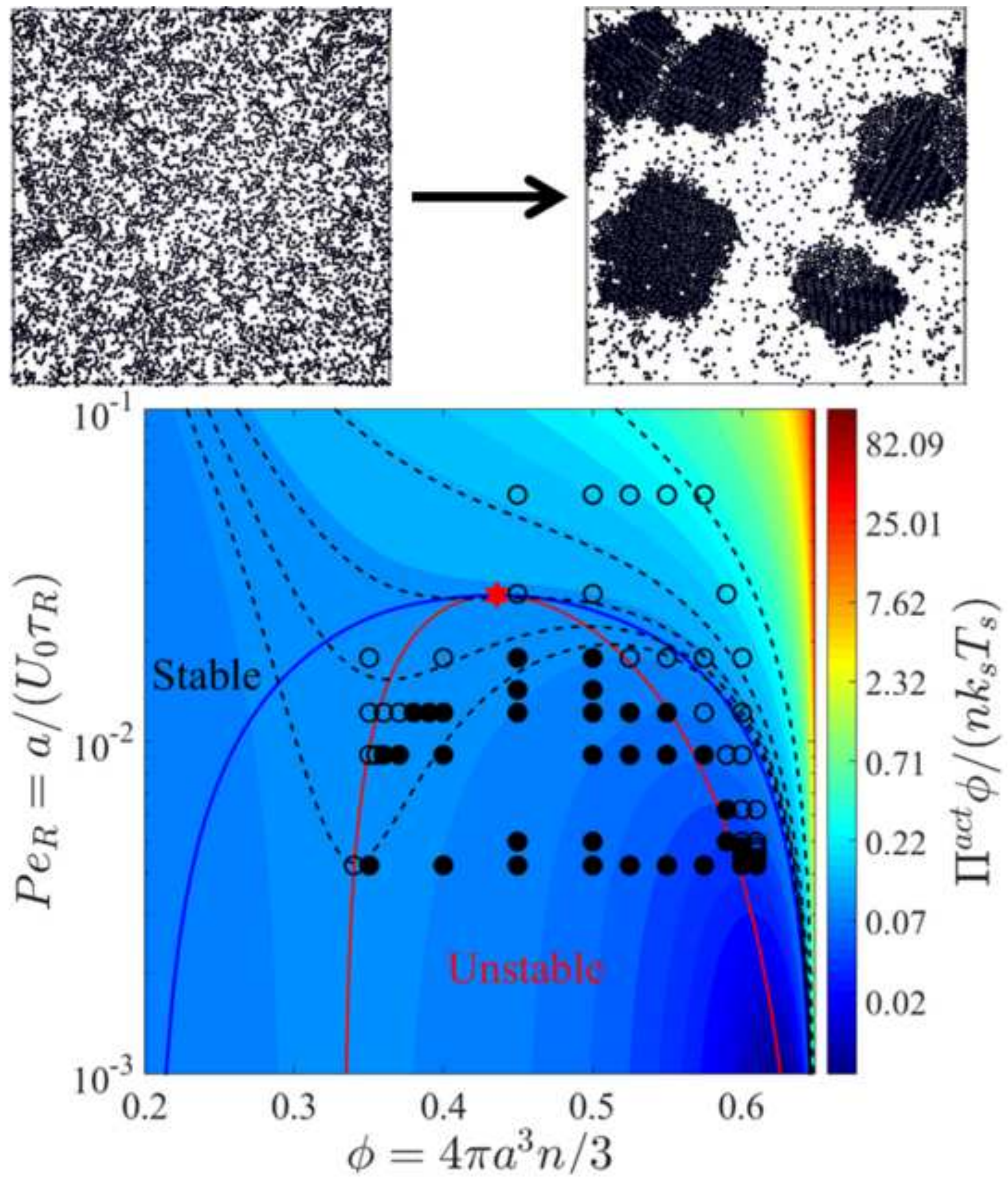

